
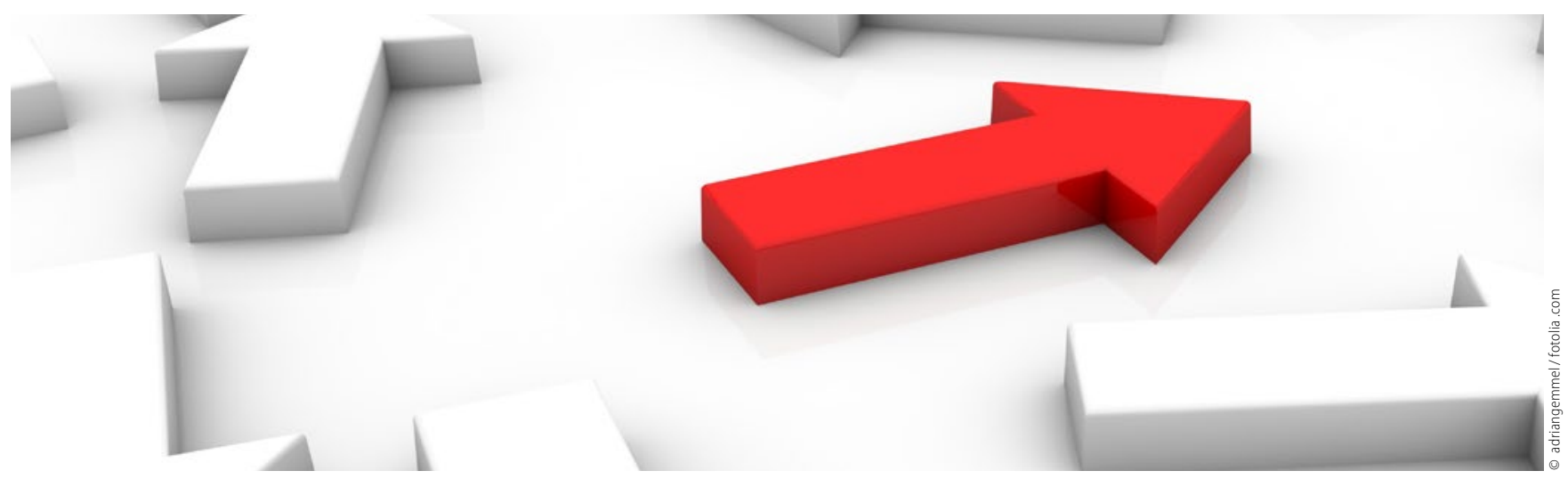

Ergänzungsleistungen - Der gangbare Weg

\title{
Die Totalprothese als Kassenleistung
}

\begin{abstract}
Trotz der Fortschritte der Zahnmedizin, gerade in der Prophylaxe, gibt es immer noch Mitmenschen, die vollkommen zahnlos sind - aus welchem Grund auch immer. Gerade in der Totalprothetik hat sich bei der Entwicklung der Materialien und Möglichkeiten einiges getan. Was aber sind die Leistungen, die die gesetzliche Krankenkasse (GKV) übernimmt?
\end{abstract}

Unter den Nummern 97a und b des zahnärztlichen Bewertungsmaßstabes (BEMA) werden Totalprothesen für den Oberkiefer und Unterkiefer beim gesetzlich versicherten Patienten abgerechnet. Dazu gehören: anatomische Abformung (auch des Gegenkiefers), Bissnahme, Farbbestimmung, Einprobe, Eingliedern, Nachbehandlung. Funktionsabformungen mit individuellem Löffel im Oberkiefer oder Unterkiefer können mit den BEMA - Nummern 98b (OK)oder 98c (UK) abgerechnet werden.

Diese Prothesen können auch als Immediat- oder Interimsversorgung dienen. Hierbei ist darauf zu achten, was die Endversorgung ist. Eine Immediatversorgung soll unterfüttert, auch mehrfach die zukünftige Versorgung darstellen. Eine Interimsversorgung dient zur Überbrückung der Zeit, bis zur endgültigen Versorgung.

\section{Abrechnung auch für Ausnahmefälle}

Die Abrechnungsnummern 97a und b dienen auch der Abrechnung von Coverdenture-Prothesen auf nicht mehr als drei Zähnen mit ausreichender prothetischer Wertigkeit je Kiefer in Verbindung mit Teleskopen oder Konuskronen oder Wurzelstiftkappen. In festgelegten Ausnahmefällen, zum Beispiel bei atrophiertem Kiefer, kann auch auf Implantaten eine Versorgung mit Coverdenture-Prothese nach 97 erfolgen.

Ebenfalls in Ausnahmefällen, beispielsweise ausgeprägter Torus palatinus oder Exostosen, kann eine Metallbasis unter der BEMA-Nummer 98e abgerechnet werden.

Wenn die Lagebeziehung von Unterkiefer zu Oberkiefer mit einfacheren Mitteln nicht reproduzierbar ermittelt werden kann und diese Leistung eindeutig dokumentiert wurde, kann BEMA-Nummer 98d für eine Stützstiftregistrierung abgerechnet werden.

Hinsichtlich der gesetzlich krankenversicherten Patienten ist noch einmal gesondert darauf hinzuweisen, dass funktionsana- lytische und funktionstherapeutische Leistungen nach $\$ 28$ Abs. 2 Satz 8 SGB V und Ziffer 33 der Zahnersatz-Richtlinien nicht zur vertragszahnärztlichen Behandlung im Rahmen der gesetzlichen Krankenversicherung gehören. Die Berechnung dieser Leistungen erfolgt bei GKV-Versicherten auf der Basis der Gebührenordnung für Zahnärzte (GOZ) im Rahmen der Vereinbarungen gemäß $₫ 4$ Abs. 5 BMV-Z beziehungsweise $₫ 7$ Abs. 7 EKVZ.

\section{Weitere Leistungen nicht per Kassenabrechnung}

Alle weiteren Leistungen, die im Einzelfall aus Gründen, die im Patientenwunsch liegen, notwendig werden, sind nicht über die gesetzliche Krankenkasse abrechenbar. Dies gilt auch für die Laborleistungen. Wünscht ein Patient besondere Zähne oder die Nachbearbeitung konfektionierter Zähne, möchte er eine ganz besondere Zahnstellung oder einen besonderen Prothesenkunststoff, so müssen diese Leistungen, wie im Artikel von Dr. Dr. Alexander Raff beschrieben, privat vereinbart werden. So kann sich jeder Patient die Prothese zusammenstellen, die er haben möchte und bekommt von der Krankenkasse den Zuschuss, der festgesetzt ist.

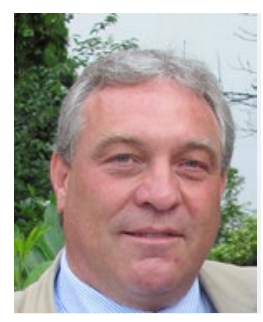

Dr. Christian Öttl

Mitglied im FVDZ-Bundesvorstand 